\title{
Use of bacteriophage in the treatment of experimental animal bacteremia from imipenem-resistant Pseudomonas aeruginosa
}

\author{
JING WANG ${ }^{1 *}$, BEI HU ${ }^{2 *}$, MINCHAO XU ${ }^{2}$, QUN YAN ${ }^{2}$, SHUANGYOU LIU $^{2}, X_{\text {XHUI ZHU }}^{3}$,

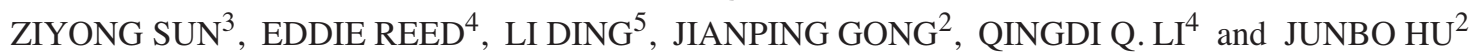 \\ ${ }^{1}$ Department of Immunology, Tongji Medical College; ${ }^{2}$ Center for Molecular Medicine and Department of Surgery, \\ ${ }^{3}$ Department of Laboratory Medicine, Tongji Hospital, Central China University of Science and Technology, Wuhan 430030, \\ P.R. China; ${ }^{4}$ Mary Babb Randolph Cancer Center and Department of Microbiology, Immunology and Cell Biology, \\ West Virginia University Health Sciences Center, Morgantown, WV 26506; ${ }^{5}$ Laboratory of Clinical Infectious Diseases, \\ National Institute of Allergy and Infectious Diseases, National Institutes of Health, Bethesda, MD 20892, USA
}

Received September 20, 2005; Accepted November 4, 2005

\begin{abstract}
The emergence of antibiotic-resistant bacterial strains still remains a significant problem for antimicrobial chemotherapy in the clinic. Bacterial viruses (bacteriophages or phages) have been suggested to be used as alternative therapeutic agents for bacterial infections. However, the efficacy of phage therapy in treating drug-resistant infections in humans is uncertain. Therefore in the present study, we examined the effectiveness of phages in the treatment of imipenem-resistant Pseudomonas aeruginosa (IMPR-Pa) infection in an experimental mouse model. Twenty-nine strains of phage were isolated from our hospital sewage, and phage ØA392 was representatively used for all testing because it has lytic activity against a wide range of clinical isolates of IMPR-Pa. We found that intraperitoneal (i.p.) injections of one IMPR-Pa strain $\left(3 \times 10^{7} \mathrm{CFU}\right)$ caused bacteremia and all mice died within 24 h. A single i.p. inoculation of the phage strain $(\mathrm{MOI} \geq 0.01)$ at up to $60 \mathrm{~min}$ after the bacterial challenge was sufficient to rescue $100 \%$ of the animals. This lifesaving effect coincided with the rapid appearance of ØA392 in the circulation (within $2 \mathrm{~h}$ after i.p. injection), which remained at substantially higher levels for up to $48 \mathrm{~h}$ until the bacteria were eradicated. However, the survival rates of the mice dropped to approximately $50 \%$ and $20 \%$ when the same dose of this purified phage preparation was administered at $180 \mathrm{~min}$ and $360 \mathrm{~min}$, respectively, after
\end{abstract}

Correspondence to: Dr Junbo Hu or Dr Jianping Gong, Department of Surgery, Tongji Hospital, Tongji Medical College, Wuhan 430030, P.R. China

E-mail: jbhu@tjh.tjmu.edu.cn jpgong@tjh.tjmu.edu.cn

Dr Qingdi Q. Li, 216 Watkins Pond Boulevard, Rockville, MD 20850-5622, USA

E-mail: qli@hsc.wvu.edu

${ }^{*}$ Contributed equally

Key words: phage therapy, animal model, mouse, P. aeruginosa, bacteremia, drug resistance, imipenem resistance
IMPR-Pa infections. In addition, we demonstrated that the ability of this phage to rescue bacteremic animals was due to the functional capabilities of the phage and not to a nonspecific immune effect. The protection from death occurred only in animals inoculated with bacteria-specific virulent phage strains. When the heat-inactivated phages were used, the survival rate of the infected mice was dramatically reduced to $20 \%$ or lower. Moreover, the levels of the antibody against the phage were not significantly changed at the time when the bacteremic animals were protected by the active phages. Finally, our observations revealed that the inoculation of the mice with high-doses of ØA392 alone produced no adverse effects attributable to the phage. These data indicate that phages can save animals from pernicious $P$. aeruginosa infections and suggest that phage therapy may be potentially used as a stand-alone therapy for patients with IMPR-Pa infections.

\section{Introduction}

Pathogenic bacteria that are resistant to a variety of antibiotics (1-3) remain a major problem in clinical practice in China and the United States, as well as the rest of the world. There are quite a few different kinds of drug-resistant bacteria that have attracted attention, some of the most common being PRSP (penicillin resistant Streptococcus pneumoniae), VRE (vancomycin resistant Enterococcus), MRSA (methicillin resistant Staphylococcus aureus), MDR-Pa (multiple drugresistant Pseudomonas aeruginosa), VRSA (vancomycin resistant Staphylococcus aureus), and extended spectrum Blactamase enzymes (ESBLs)-producing bacteria. Although new antibiotics are being developed constantly, bacteria that are resistant to these antibiotics will emerge sooner or later, which severely reduces the efficacy of conventional antibiotic chemotherapy. Therefore, alternative remedial strategies, such as biologic therapeutics or other novel therapeutics, need to be developed to combat bacterial infections and treat a broad array of infectious diseases.

Bacteriophages (phages) are viruses that infect and destroy bacteria. Phage therapy is a method of harnessing phages as therapeutic agents for the treatment of bacterial infectious 
diseases. As early as the 1920 s, substantial work was performed in this field (4-6). Despite these great efforts, the use of phages as therapeutic agents was generally abandoned in the Western world soon after the discovery of antibiotics in the 1940s. The feasibility of phage therapy is still under investigation. The main reasons may include: i) the host range of the phages used was too narrow; ii) the large load of endotoxins and exotoxins in the bacterial debris in the poorly-purified phage lysates; iii) the pharmacokinetics of phages in vivo was not very clear, even the understanding of phage biology was limited; iv) the immune response of the body to phages; v) the lysogenesis mutation of the lytic phages used; and vi) the resistance of bacteria to phages. Moreover, the studies of early investigators were mostly uncontrolled or poorly designed. Intriguingly, in several Eastern European countries, the former Soviet Union and France, phage therapy is maintained till now and a lot of successful experience has been acquired. With the emergence of drug-resistant bacteria worldwide, phage therapy as an alternative approach has been reappraised (7). Although a series of rigorous investigations have been undertaken since the 1980s (8-12), very limited studies have been reported (12-14) that confirmed the authenticity of phage therapy for Pseudomonas aeruginosa-caused diseases.

Pseudomonas aeruginosa (P. aeruginosa) is an important human pathogen implicated in severe clinical infections such as lethal sepsis and nosocomial infections, like pneumonia and urinary tract infection, and is associated with severe sequelae and mortality. Multiple drug-resistant $P$. aeruginosa isolates are more and more commonly seen in Chinese hospitals due to the excessive and often unnecessary use of antibiotics in humans, which has probably advanced the emergence and spread of the drug-resistant pathogen. Now $P$. aeruginosa has become the primary pathogen of nosocomial infection according to the report of Chinese National Pathogen Resistance Surveillance (CNPRS). The report also indicated that the susceptibility of $P$. aeruginosa to the 11 main antibiotics are declining, among them the susceptibility to imipenem and ceftazidime, declined from $96 \%$ and $92 \%$ to $75 \%$ and $79 \%$, respectively, from 1994 to 2001 . Imipenem is the principal antibiotic used to control Gram-negative bacterial infections, and there is no other choice of antibiotics if bacteria are resistant to imipenem. Moreover, when $P$. aeruginosa are resistant to an antibiotic such as imipenem in the clinic, they are usually resistant to other antibiotics as well. Hence, imipenem-resistant $P$. aeruginosa (IMPR-Pa) infection is one of the most intractable clinical problems.

The aim of the present study was to investigate the effectivity and feasibility of phage therapy as an alternative regimen for the treatment of drug-resistant bacterial infections. We report the isolation of bacteriophages that are safe and effective as bactericidal agents for animals with systemic lethal IMPR-Pa infections.

\section{Materials and methods}

Culture media and reagents. LB media and SM buffer were prepared according to Sambrook et al (15) TSBM is TSB medium supplemented with $20 \mathrm{mM} \mathrm{MgCl}_{2}$. $\mathrm{PEG} / \mathrm{NaCl}$ is $20 \%$ PEG-8000 (w/v) supplemented with $2.5 \mathrm{M} \mathrm{NaCl}$. LB- based solid medium containing $1.5 \%$ agar or $0.7 \%$ agarose was used for the lower or upper layers, respectively. Hind-III and $\lambda$ Hind-III digest markers were purchased from New England and Takara Co., respectively. DNAse I, RNAse A and Proteinase K were purchased from Becbo, Sigma, and Amresco Co., respectively.

Bacterial strains. The bacterial strains used in our study included 30 IMPR-Pa strains, which were all resist to imipenem. The IMPR-Pa samples were derived from clinical specimens obtained from patients at Tongji Hospital. In the present study, IMPR-Pa strain A392 served as the propagation host and experimental target of our specific $P$. aeruginosa phage ØA392 (see below), unless otherwise stated, because A392 is sensitive to most of the phages isolated, including ØA392. Three other IMPR-Pa strains were utilized for some animal experiments. Bacterial growth was monitored by measuring turbidity with a UV-2000 spectrophotometer. A $0.5 \mathrm{OD}_{600}$ value was assumed to be equivalent to $2 \times 10^{8} P$. aeruginosa cells $/ \mathrm{ml}$. This conversion formula was based on a previously standardized correlation between turbidity and bacterial cell numbers counted directly by quantitative plating.

Isolation and purification of phage strains. The IMPR-Pa strains mentioned above were used as the host to isolate phage strains from the raw sewage from the Sewage Treatment Center of Tongji Hospital. The isolations, performed similarly but individually for each phage strain according to Biswas et al (16), were accomplished by adding salt $(58 \mathrm{~g}$ of $\mathrm{NaCl})$ to 11 of sewage followed by centrifugation at $10,000 \mathrm{x}$ g for $10 \mathrm{~min}$. The supernatant was decanted into a separate container and mixed with polyethylene glycol (PEG; molecular weight, $8,000)$ to provide a final concentration of PEG of $10 \%(\mathrm{w} / \mathrm{v})$. The PEG-containing supernatant was precipitated overnight at $4^{\circ} \mathrm{C}$ and centrifuged at $12,000 \mathrm{x}$ g for $20 \mathrm{~min}$. The resulting precipitate was dissolved in $5 \mathrm{ml}$ of phage dilution buffer (SM) and extracted once with an equal volume of chloroform. An aliquot $(300 \mu 1)$ of this processed sewage was mixed with $200 \mu \mathrm{l}$ of an overnight culture of IMPR-Pa strains, incubated at $37^{\circ} \mathrm{C}$ for $20 \mathrm{~min}$, mixed with $3 \mathrm{ml}$ of molten top agar $(0.7 \%$ agarose $)$ at $50^{\circ} \mathrm{C}$, and poured on $\mathrm{LB}$ agar plates $(1.5 \%$ agar; see above). Plates were incubated overnight at $37^{\circ} \mathrm{C}$. Phage plaques were harvested from the plate and single plaques were purified three times on host strains by the standard procedures described by Sambrook et al (15).

Large-scale amplification and purification of phage particles. Phage ØA392 was purified essentially according to the procedure described by Sambrook et al (15) A392 host cells suspended at $2 \times 10^{8}$ cells $/ \mathrm{ml}$ in $20 \mathrm{ml} \mathrm{LB}$ medium were exposed to a crude preparation of $\varnothing \mathrm{A} 392$ at an MOI of 0.01 and were vigorously shaken for $4-5 \mathrm{~h}$ at $37^{\circ} \mathrm{C}$, resulting in complete lysis of the bacteria. After treatment with $1 \%$ chloroform at $37^{\circ} \mathrm{C}$ for $10 \mathrm{~min}$, and with $1 \mu \mathrm{g} / \mathrm{ml}$ DNase I and $1 \mu \mathrm{g} / \mathrm{ml} \mathrm{RNase}$ A for $30 \mathrm{~min}$ at $37^{\circ} \mathrm{C}$, the culture fluid was centrifuged at $12,000 \mathrm{x}$ g for $10 \mathrm{~min}$ at $4^{\circ} \mathrm{C}$ to remove cell debris. PEG/NaCl was added to the supernatant to final concentrations of $1 / 6$ (v/v) and kept at $4{ }^{\circ} \mathrm{C}$ overnight. The resultant precipitate containing the phage particles was collected by centrifugation 
at $10,000 \mathrm{x} \mathrm{g}$ for $20 \mathrm{~min}$ at $4^{\circ} \mathrm{C}$, resuspended in $500 \mu 1 \mathrm{SM}$ buffer, the phage suspension then was placed on top of a discontinuous $\mathrm{CsCl}$ gradient $(\varrho=1.3, \varrho=1.5$, and $\varrho=1.7)$ and centrifuged at 25,000 x g for $2.5 \mathrm{~h}$ at $4^{\circ} \mathrm{C}$ (Beckman, SW41 rotor). The phage band was collected and dialyzed against $10 \mathrm{mM}$ saline that contained $50 \mathrm{mM}$ Tris- $\mathrm{HCl}(\mathrm{pH} \mathrm{8.0)}$ and $10 \mathrm{mM} \mathrm{MgCl}{ }_{2}$ for $2 \mathrm{~h}$ at $4^{\circ} \mathrm{C}$. CsCl-gradient separation and dialysis $(1 \mathrm{~h})$ were repeated. The purified phage suspension was divided into aliquots, and stored at $4^{\circ} \mathrm{C}$ until used. The samples were appropriately diluted with LB just before use for infections. The titers (PFU/ml) of the purified samples were determined by inoculating them into bacterial strain A392.

Biological characterization of phage ØA392. The adsorption rate, latent period, and burst size of ØA392 were determined essentially according to the method of Adams (17). All incubations for the analyses were carried out in LB medium at $37^{\circ} \mathrm{C}$. In brief, for examination of the adsorption rate, ØA392 $\left(5 \times 10^{9} \mathrm{PFU}\right)$ were mixed with $\mathrm{A} 392$ cells $\left(5 \times 10^{7} \mathrm{CFU}\right)$, and the number of free infectious phage virions was measured in the phage-cell mixture diluted 1,000 times. To determine the latent period and burst size, A392 cells $\left(5 \times 10^{6} / \mathrm{ml}\right)$ were

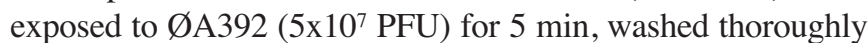
with cold TSBM medium to remove unbound phages, and then resuspended in fresh medium. An aliquot of the cell suspension was harvested regularly during incubation at $37^{\circ} \mathrm{C}$ to be titrated for newly produced phages, including both released and cell-associated phages, on a lawn of A392.

Screening phages with broad host range. Diluted bacterial strains were used to made lawns on solid culture. And the back of the plate was divided into 16-20 panes. All the panes were marked with the name of corresponding phages. Then phages were dropped onto it and the plates were cultured at $37^{\circ} \mathrm{C}$ for $12-16 \mathrm{~h}$.

Animal experiments. IMPR-Pa A392 was used to infect mice because it was susceptive to most phages isolated, while ØA392 was used to rescue mice as it has the widest host range according to the results of screening.

Varying numbers of bacterial cells suspended in $0.5 \mathrm{ml}$ saline were injected into the peritoneal cavities of mice through one side of the abdomen, and purified phage suspensions in $1 \mathrm{ml} \mathrm{LB}$ medium were injected through the other side. As controls, equal volumes of saline or LB medium alone were injected intraperitoneally on all test occasions. The test animals were observed for 1 week to 20 days.

Establishing the MLD in the mouse model. For infection experiments, 6- to 8-week-old BALB/c female mice (body weight, $\sim 20 \mathrm{~g}$ ) were used. Preparation of the infecting bacteria was as follows: IMPR-Pa cells were grown in 100 $\mathrm{ml} \mathrm{LB}$ medium at $37^{\circ} \mathrm{C}$ and were centrifuged at $8,000 \mathrm{x}$ g for $5 \mathrm{~min}$ at the early stationary phase $\left(\sim 0.5 \mathrm{OD}_{600}\right.$ value $)$. The cell pellet was washed with $100 \mathrm{ml}$ saline, centrifuged again under the same conditions, and finally resuspended in $5 \mathrm{ml}$ saline. After appropriate dilution, turbidity was measured to determine bacterial cell numbers, as described above. To determine the minimum lethal dose (MLD), serial dilutions of A392 were injected intraperitoneally (i.p.) into mice in $500 \mu \mathrm{l}$ aliquots. The animals were observed for $>100 \mathrm{~h}$.

Treatment with phage. The efficacy of phage therapy was evaluated in two separate experiments using the IMPR-Pa bacteremia mouse model. The first examined the effect of phage dose on the ability of phage to rescue mice from IMPR-Pa bacteremia. The second studied the effect on the outcome of delaying treatment for various periods. In the dose-ranging study, 11 groups of mice ( 5 mice in each) were challenged by i.p. injection of the MLD of A392. Each of these groups was treated with a single injection of phage ØA392, administered i.p. immediately after the bacterial challenge at MOI as $0,10^{-4}, 10^{-3}, 10^{-2}, 0.1,1,10,100$, or 200.

In the delayed-treatment study, treatment (a single injection of phage at the highest dose) was initiated at 0,15 , $30,45,60,180$, and $360 \mathrm{~min}$ after the bacterial challenge with the MLD of A392. The state of the health of these animals was also monitored for 20 days.

Effects of heat-inactivated phage. An experiment was performed to determine whether phage rescue of mice with IMPR-Pa bacteremia requires phage that can grow on the bacterial host or whether phage rescue might be associated with a non-specific immune activation response. A sample of ØA392 with a titer of $6 \times 10^{9} \mathrm{PFU} / \mathrm{ml}$ was heat inactivated by incubation at $100^{\circ} \mathrm{C}$. Phage that had been heated for a total of $20 \mathrm{~min}$, at which time no viable phage was detectable, was used to determine whether phage rescue of mice with IMPR$\mathrm{Pa}$ bacteremia requires functional phage or whether the rescue might be associated with non-specific immune activation. The mice in this study were divided into 3 groups of 5 each. All of the mice were challenged by i.p. injection of the MLD of A392. One group was treated with a single i.p. injection of $6 \times 10^{9}$ PFU of ØA392 immediately after the bacterial challenge. The second group was treated by i.p. injection with $6 \times 10^{9} \mathrm{PFU}$ of heat-inactivated ØA392 particles immediately after the bacterial challenge. The third group, a control, received a single injection of LB medium instead of phage, administered i.p. immediately after the bacterial challenge.

The pharmacokinetics in vivo of A392. Target bacterium ( $3 \times 10^{7}$ cells) and/or ØA392 (MOI, 200) were injected intraperitoneally into 3 groups of mice, each of which consisted of 5 mice. Approximately $0.1 \mathrm{ml}$ of blood was taken by puncturing the orbital plexus of test mice with a capillary tube and mixed immediately with $50 \mu 1$ heparin $(1,000 \mathrm{U} / \mathrm{ml})$. After the heparinized blood was diluted with saline, colonyforming units of A392 were measured on LB plates, and plaque-forming units of ØA392 were measured by use of A392 as the host on LB plates. Blood samples were also collected from untreated mice, to ensure that mice used in the experiments were free of naturally or accidentally contaminating phage or bacteria.

The effect of mixed phage. Phage ØA392 and phage Ø1093 were mixed together to make a 'cocktail' phage mixture. Four IMPR-Pa strains were selected to infect 4 groups of mice: IMPR-Pa 9747, 9994, 1613, and 9915. Phage mixture displayed no plaque-forming activity on 9994 in vitro, while 
(A)

(B)

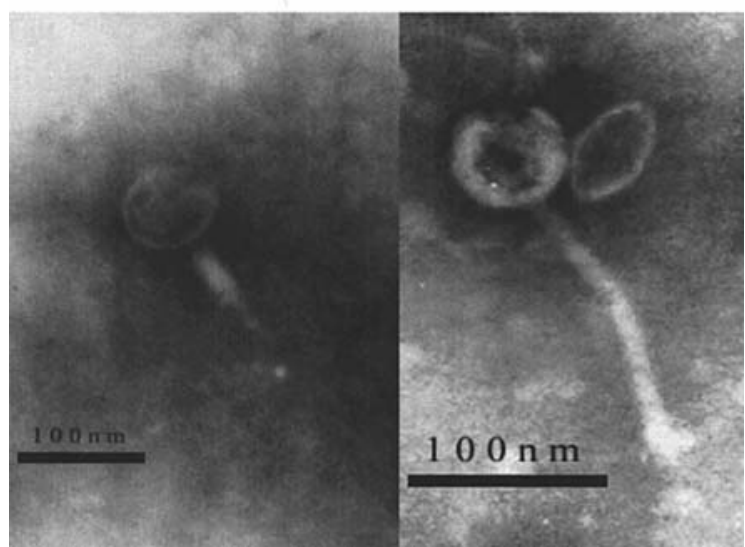

Figure 1. Electron micrographs of phage ØA392 (A) and phage Ø1093 (B). Purified phage samples in SM were spread on a copper grid coated with Formvar and negatively stained with $2 \%$ PTA (pH 7.0). Electron micrographs were taken with a transmission electron microscope. Bar, $100 \mathrm{~nm}$.

it formed clear plaques on the other three IMPR-Pa strains. The survival rates of the mice in the 4 groups were monitored for 20 days.

Measuring the immune response to the phage. At various time points following a single i.p. injection of ØA392 (109 PFU), mouse serum was collected, and indirect enzyme-linked immunosorbent assays (ELISAs) were performed. For these assays, 2-fold serial dilutions of serum were prepared in 96-well polystyrene microtiter plates precoated with $10^{9} \mathrm{PFU}$ of ØA392. Immunoglobulin was detected with goat antimouse IgG-specific antibody conjugated to peroxidase and tetramethylbenzadine (TMB) peroxidase substrate.

Electron microscopy. Purified phage samples in SM were spread on a copper grid coated with Formvar and negatively stained with 2\% PTA (pH 7.0). Electron micrographs were taken with a transmission electron microscope (JEM 2100 C; Hitachi).

\section{Results}

Isolation and screening of P. aeruginosa specific phages with therapeutic potential. Cultures of $30 P$. aeruginosa strains (IMPR-Pa) were subjected to the spot test. Of the phage strains isolated in these experiments, one (ØA392) was found to form plaques on $75 \%$ of the IMPR-Pa clinical isolates and the other (Ø1093) was found to form plaques on $41 \%$ of the IMPR-Pa clinical isolates. And the lytic spectrum of the two phages mentioned above is complementary to each other.

Morphological, biological, and genetic characterizations of phage ØA392. As shown in Fig. 1, electron microscopy demonstrated that ØA392 has an isometrically hexagonal head, $66 \pm 2 \mathrm{~nm}$ in diameter, and a contractile tail of $116 \pm 5$ $\mathrm{nm}$ in length, while $\varnothing 1093$ has an isometrically hexagonal head, $60 \pm 2 \mathrm{~nm}$ in diameter, and a non-contractile tail of $170 \pm 5 \mathrm{~nm}$ in length. Restriction-enzyme analysis revealed

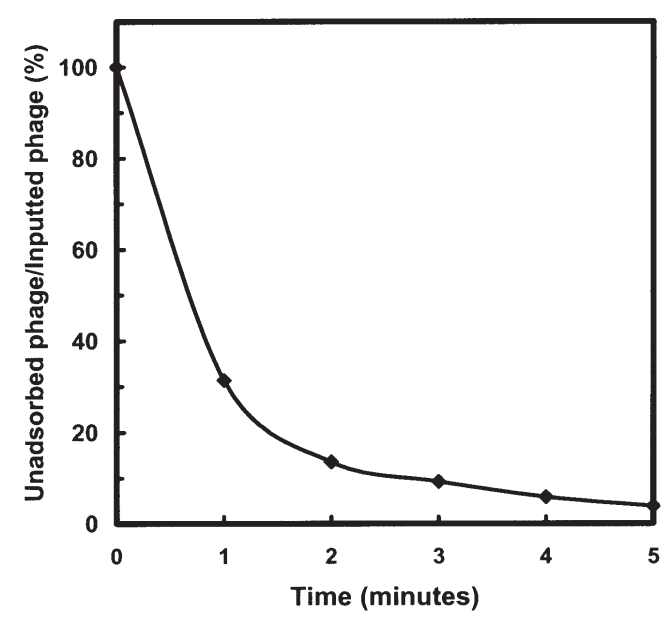

Figure 2. The adsorption rate of phage ØA392. Phages were mixed with excess $P$. aeruginosa A392 cells, and then non-adsorbed infectious phages were serially counted. Data are percentage of non-adsorbed ØA392 relative to the initial input dose of phages.

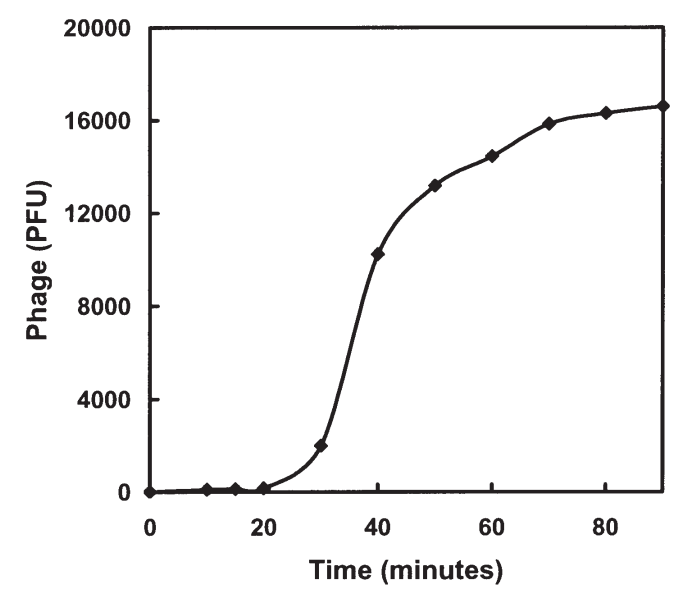

Figure 3. The one-step curve of phage ØA392. After a 5-min adsorption of ØA392 to a sufficient number of A392 cells, the ØA392 exposed bacterial cells were thoroughly washed with tryptic soy broth medium supplemented with $20 \mathrm{mM} \mathrm{MgCl}_{2}$ at $4^{\circ} \mathrm{C}$ to remove free phages and then were resuspended in fresh medium. Newly synthesized phage virions, including both released and cell-associated virions, then were measured at regular intervals during the incubation, using harvested culture samples.

that the genome DNA of ØA392 and Ø1093 are discrepant. Biological studies clarified other features of ØA392 (Figs. 2 and 3), such as the following: i) a rapid adsorption rate (when sensitive bacterial hosts were present in sufficient numbers, $>96 \%$ of ØА392 particles bound to them within $5 \mathrm{~min}$ ); ii) a short latent period ( $25 \mathrm{~min})$; and iii) a relatively large burst size $(\sim 125)$.

On the evidence given above, ØA392 was considered to be a suitable candidate of the therapeutic phage for the treatment of human $P$. aeruginosa infections. Therefore, the following experiments were undertaken using ØA392 in an animal model. And the two phages (ØA392 and Ø1093) can be mixed to make a 'cocktail' for extensive applications. 


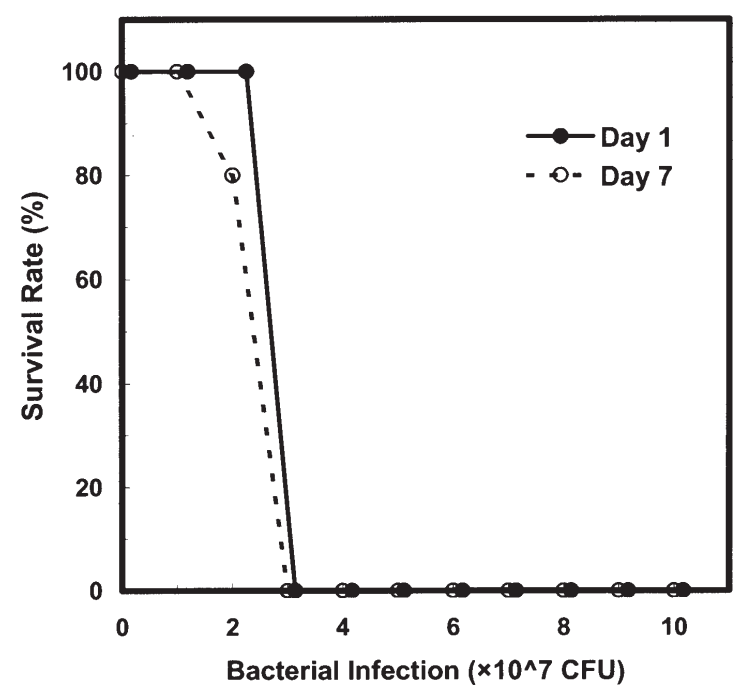

Figure 4. Determination of the challenge dose of $P$. aeruginosa lethal to mice. Serially diluted suspensions of $P$. aeruginosa A392 cells were injected intraperitoneally into mice, and mouse fatalities were observed. White and black circles represent the survival rates 1 and 7 days after injection, respectively. In the control experiment, denoted ' 0 ' on the horizontal axis, only $0.5 \mathrm{ml}$ of saline was injected.

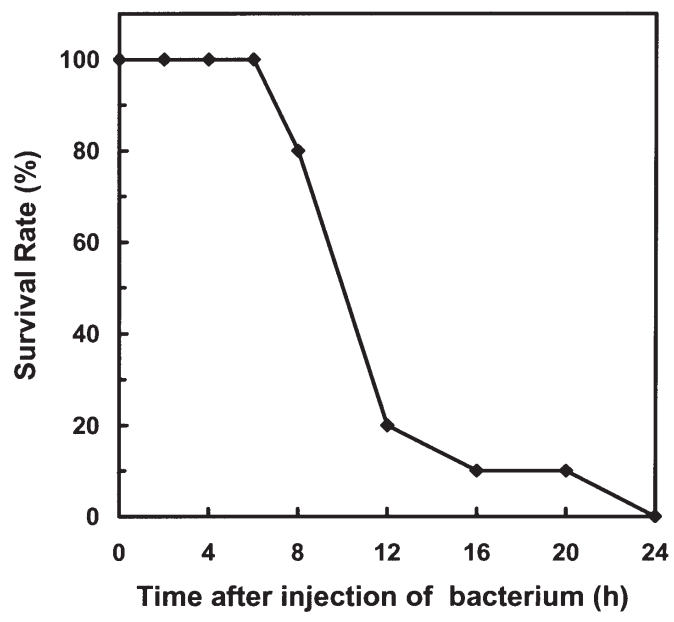

Figure 5. A precise time-chase analysis of $P$. aeruginosa-induced killing of mice. Ten mice were each inoculated with a fixed number of $P$. aeruginosa A392 cells $\left(3 \times 10^{7}\right)$ and their mortality was traced for up to $24 \mathrm{~h}$. Number of surviving mice dropped suddenly $6 \mathrm{~h}$ after bacterial challenge, and death was preceded by obvious bacteremia.

Mouse model of P. aeruginosa induced disease. The dose of $P$. aeruginosa lethal to mice was determined by injecting mice with varying numbers of strain A392, ranging from $1 \times 10^{7}$ to $1 \times 10^{8}$ cells per dose (Fig. 4). Intraperitoneal (i.p.) injections of $1 \times 10^{7}-2 \times 10^{7}$ A392 did not reduce the survival rate of mice during the subsequent 7 -day observation period. By contrast, injections of $3 \times 10^{7}-1 \times 10^{8}$ cells lowered the survival rate in a dose-dependent manner. Because the injection of $3 \times 10^{7}$ A 392 cells was fatal in $100 \%$ of mice within $24 \mathrm{~h}$, this level of challenge was considered to be optimal for observing the phage effect on bacteria-induced lethality. Therefore, the dose of $P$. aeruginosa was fixed at $3 \times 10^{7}$ cells

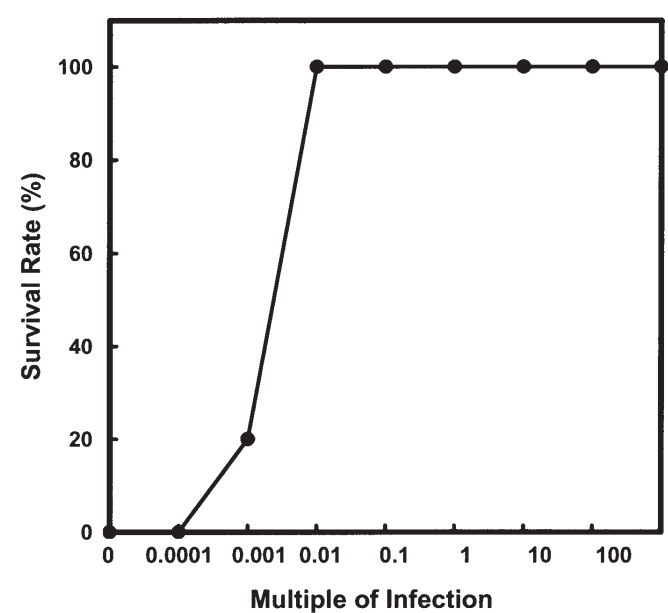

Figure 6. Dose effect of phage ØA392 in rescuing mice from lethal imipenemresistant $P$. aeruginosa bacteremia. After injection of $P$. aeruginosa A392 $\left(3 \times 10^{7}\right)$ cells, ØA392 were injected into the mouse peritoneal cavity at various MOIs, and the fate of the mouse was observed. Mice injected only with LB broth, which was used to prepare the phage suspensions, served as controls and are represented by circles at a MOI of 0 . There is a statistically significant difference in survival rates between mice treated with ØA392 at a $\mathrm{MOI} \geq 0.01$ and untreated control mice $(\mathrm{P}<0.001)$.

throughout the following experiments. A more precise timechase analysis showed that intraperitoneal inoculation of $3 \times 10^{7}$ A392 cells killed most mice between 6 and $14 \mathrm{~h}$ after injection of bacteria, with associated preceding bacteremia (Fig. 5). Dissection of mice that died from bacterial infection $6 \mathrm{~h}$ after injection revealed severe systemic congestion, with splenomegaly and acute ascites (data not shown). As seen in Fig. 5, all mice inoculated i.p. with the MLD $\left(3 \times 10^{7} \mathrm{CFU}\right)$ of the clinical isolate IMPR-Pa strain A392 died within $24 \mathrm{~h}$.

Ability of the phage preparation to rescue mice from bacteremia. A single dose of phage ØA392 was administered immediately after the challenge with the MLD of bacteria. By $24 \mathrm{~h}$ a dose effect on the state of health of the infected animals was clearly visible. At higher doses of the phage, which achieved multiplicities of infection (MOI) of 0.01-200, $100 \%$ of the animals survived, and only mild lethargy was seen (in the first $24 \mathrm{~h}$ ). As the phage dose decreased, resulting in lower multiplicities of infection (0.0001 and $0.001)$, the animals became critically ill, with survival rates of 0 and 20\%, respectively, at day 6 and beyond (Fig. 6). All of the mice that were alive and healthy at day 6 remained so for an additional 20 days, at which point the experiment was terminated.

Temporal sequence and in vivo distribution of phage ØA392 and $P$. aeruginosa. Purified phage ØA392 was administered intraperitoneally to mice at different times (up to $360 \mathrm{~min}$ ) after A392 injection (Fig. 6). Although ØA392 administration proved to be effective at MOIs ranging widely from 0.01 to 200 (Fig. 6), the highest MOI of 200 was applied in this and subsequent investigations (see below), under the assumption that patients were in an extreme situation of systemic $P$. aeruginosa infection such as sepsis. After $6 \mathrm{~h}$, most mice treated with phages at any time point were still alive, whereas 

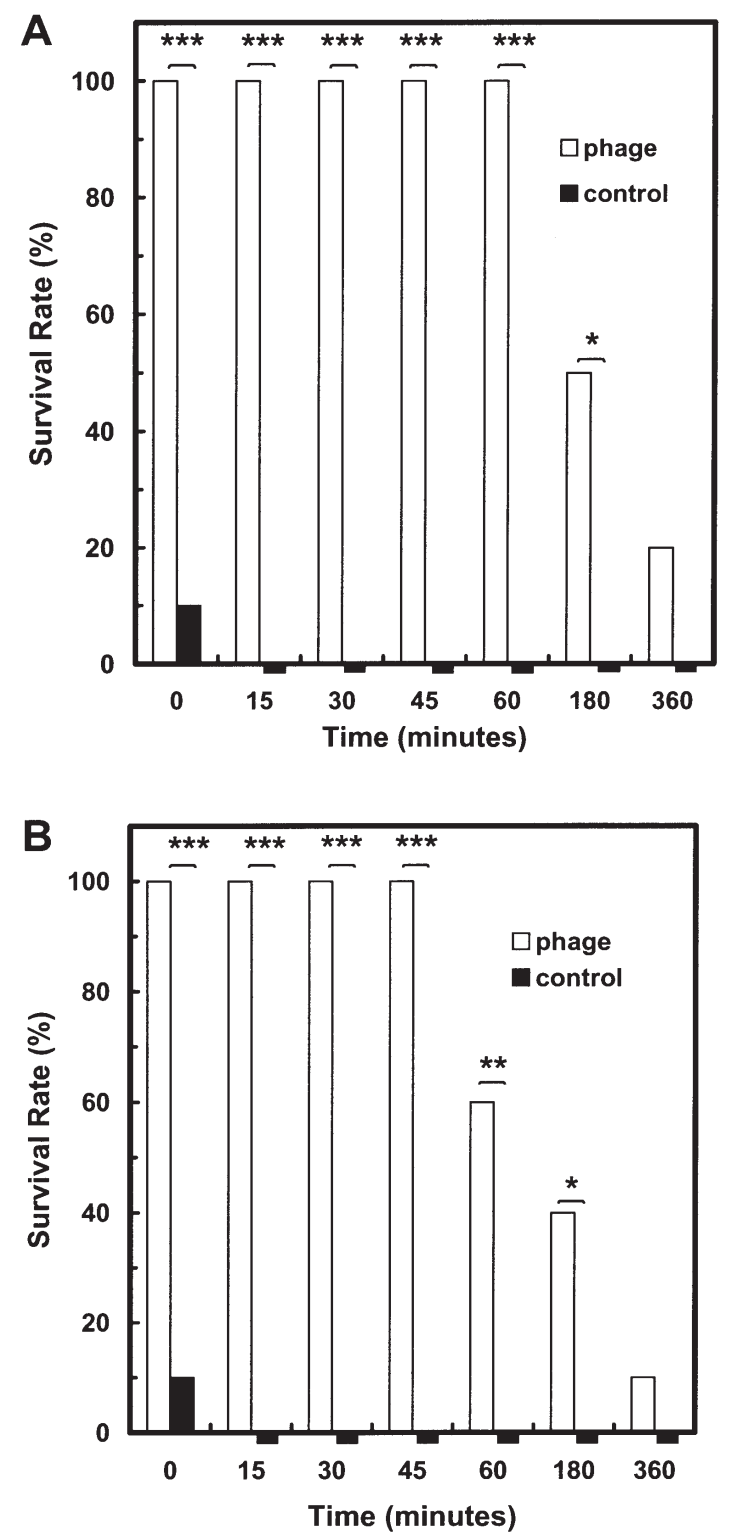

Figure 7. Protective effects with delayed administration of phage ØA392. Purified ØA392 (MOI, 200) were administered to 5 mice at the various time intervals indicated, after challenge with $P$. aeruginosa A392 ( $3 \times 10^{7}$ cells). As a control, $0.5 \mathrm{ml}$ of phage-free LB was injected into mice. Survival rates were determined after 1 (A) and 7 (B) days. White and black columns represent the phage-treated and -untreated mouse groups, respectively. Asterisks indicate statistically significant differences compared with that of the controls: ${ }^{*} \mathrm{P}<0.05,{ }^{* *} \mathrm{P}<0.01$, and ${ }^{* * *} \mathrm{P}<0.001$.

$80-100 \%$ of mice not treated with phages were dead. The therapeutic efficacy of ØA392 was even discernible in mice treated $180 \mathrm{~min}$ after injection with bacteria, when all the control mice injected with A392-only already exhibited signs of physical deterioration, such as reduced activity and ruffled hair. Although a few ØA392-treated mice died during the following 6 days, the survival rates among mice treated with phages at any time point were always significantly higher than those of the untreated controls $(\mathrm{P}<0.001)$ (Fig. 7).

On the basis of the above results, the in vivo dynamics of the bacteria and phages were investigated in detail using A392 as the target cell. Mice were injected intraperitoneally with $3 \times 10^{7}$ A392 alone, with A392 plus ØA392 (6x109 PFU),

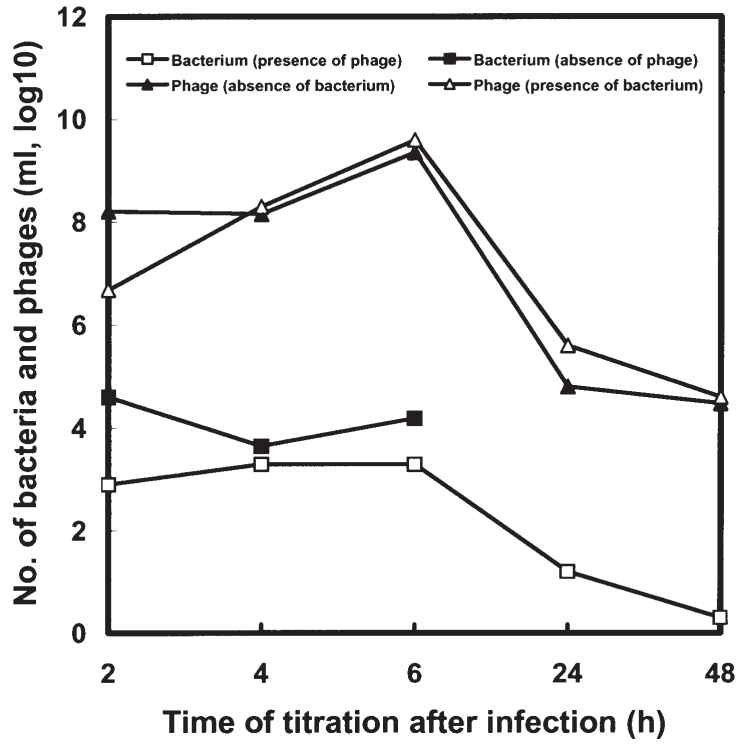

Figure 8. Rapid appearance of phage ØA392 in circulation. Target bacteria (3×107 cells) and/or ØA392 (MOI, 200) were injected intraperitoneally into 4 groups of mice, each of which consisted of 5 mice. A392 was used as the target in this experiment. A peripheral blood sample was taken from 1 mouse from each group 2, 4, 6, 24 and $48 \mathrm{~h}$ after injection and was titrated to estimate the numbers of phages and bacteria in the circulation. Black and white triangles represent plaque-forming units of ØA392 in the presence and absence of A392, respectively. Black and white squares represent colonyforming units of the host bacteria with and without the administration of phage, respectively. All mice injected with only A392 died during the observation period within $6 \mathrm{~h}$ of injection.

or with ØA392 alone, respectively. Bacteremia occurred within $2 \mathrm{~h}$ and then persisted in the A392-injected mice, regardless of phage treatment (Fig. 8). However, the bacterial loads in the blood were significantly lower in ØA392-treated mice than in untreated mice throughout our observations $(\mathrm{P}<0.05)$ (Fig. 8). Compatible with the result shown in Fig. 8, all mice injected only with A392 died within $12 \mathrm{~h}$, whereas ØA392-treated mice were invariably saved, concomitantly with a subsiding of septicemia within $24 \mathrm{~h}$. However, after Ø392 was injected into the peritoneal cavity, a significant number of infectious ØA392 was readily detected 2 h later in blood specimens from both the A392-infected and uninfected mice at titers of $4.8 \times 10^{6}$ and $1.6 \times 10^{8} \mathrm{PFU} / \mathrm{ml}$, respectively. These results illustrate the in vivo kinetics, whereby intraperitoneal ØA392 was amplified by the coexisting target bacteria and migrated into the bloodstream, perhaps resulting in the systemic dissemination of the phages. Under these circumstances, the circulating ØA392 was sustained at a significant level until the target cells were eradicated, which must have counteracted the progression of bacteremia.

Effects of heat-inactivated phage. An experiment was performed to determine whether phage rescue of mice with IMPR-Pa bacteremia requires functional phage or whether phage rescue might be associated with a non-specific immune activation response. Heat-inactivated phages were used to test whether a functional phage and not a non-specific immune response is responsible for the rescue of mice inoculated with a lethal dose of $\mathrm{A} 392$. Heating at $80^{\circ} \mathrm{C}$ for 20 min decreased the phage titer by 1,000 -fold, and no viable phage was 


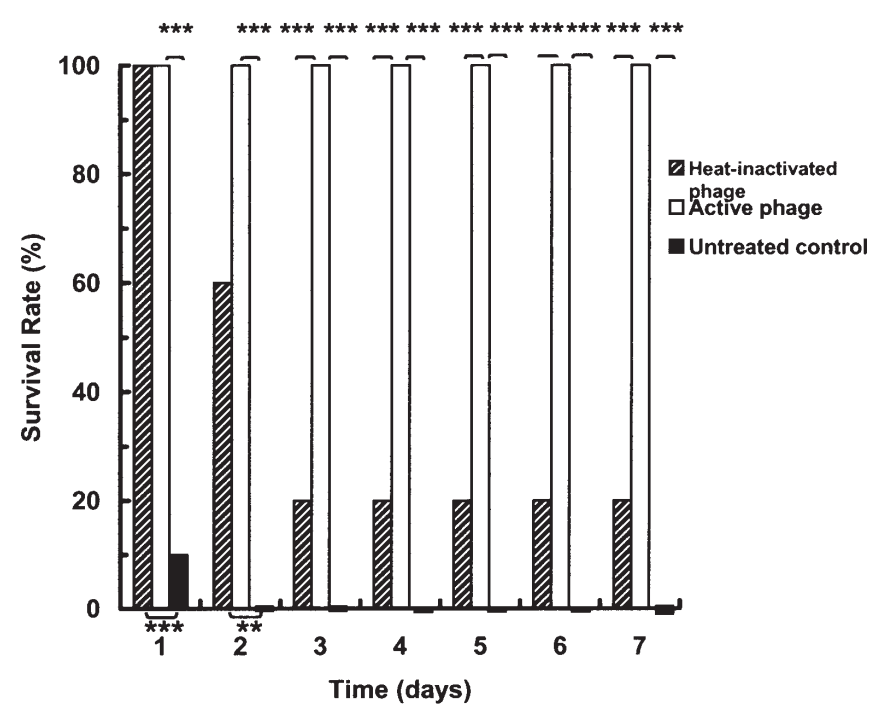

Figure 9. Comparison of the ability of active phage and non-functional heatinactivated phage to rescue bacteremic mice. One group of 5 mice was treated with functional (plaque-forming) phage, while the other two groups were treated either with no phage or heat-inactivated phage. One hundred percent of the bacteremic mice treated with functional phage survived, while only $20 \%$ of the mice in heat-inactivated groups survived, but none of the control group survived. The animals were monitored for 20 more days, and no changes in their state of health were noted.

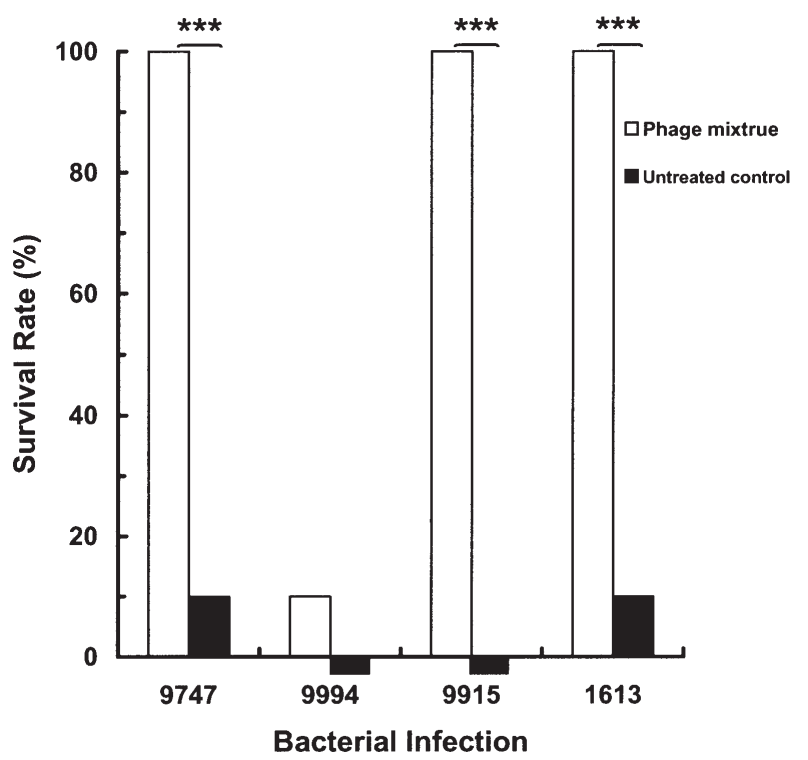

Figure 10. Effect of mixed phage on imipenem-resistant $P$. aeruginosa (IMPR-Pa) infections in mice. Phage ØA392 and phage Ø1093 were mixed together to make a 'cocktail' phage mixture. Four IMPR-Pa strains were selected to infect 4 groups of mice, and then the phage mixture was injected intraperitoneally into mice. The survival rate of the mice was monitored for 20 days. A statistically significant difference $(\mathrm{P}<0.001)$ in survival rates was observed between paired groups of mixed phage-treated mice and phageuntreated control mice, as signified by horizontal brackets with asterisks.

detected after heating at $100^{\circ} \mathrm{C}$ for $20 \mathrm{~min}$. As illustrated in Fig. 9, only mice inoculated with plaque-forming phage had enhanced survival, with $100 \%$ survival at 7 days, while only $20 \%$ of the mice injected with heat-inactivated phage survived but none of the PBS-treated control mice survived.

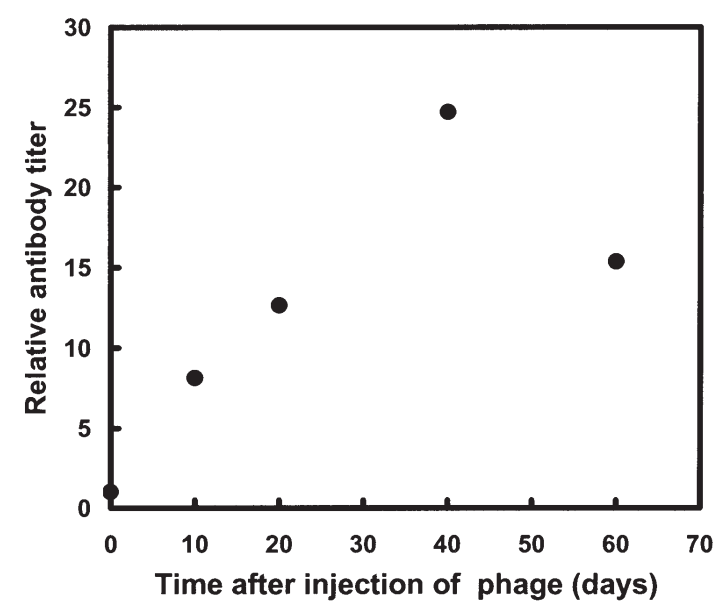

Figure 11. Antibody responses in mice to a single injection of phage ØA392. The phage was injected i.p. ( $\left.10^{9} \mathrm{PFU}\right)$. Mouse serum was collected at various time points following a single i.p. injection of ØA392, and indirect enzymelinked immunosorbent assays (ELISAs) were performed. The titers of antiØА392 IgG were detected with goat anti-mouse IgG-specific antibody and are indicated in the plot.

Effect of mixed phage. We investigated whether mixed phage is efficacious against IMPR-Pa infections. Each one of 4 clinical IMPR-Pa strains - 9747, 9915, 9994, or 1613 - was injected intraperitoneally into mice. For this experiment, phage mixture was propagated once beforehand with each corresponding IMPR-Pa strain to confirm whether it can form plaques on them or not. As shown in Fig. 10, 3 groups of mice treated with these phage mixture survived for 7 days after IMPR-Pa injection (Fig. 10), in contrast to the $>90 \%$ mortality in the phage-untreated mice, which suggests that phage mixture is potentially useful for human IMPR-Pa infections. Only 10\% mice of the other group (9994) survived (Fig. 10), which indicates that the rescue of phage is based on lytic activity.

Immune response to phage $\emptyset A 392$. After a single injection of phage ØA392, the titers of IgG raised against the phage increased above background 26-fold in 40 days (Fig. 11). However, the levels of the antibody against the phage were not significantly elevated at the time when the bacteremic animals were protected by the phage. IgG levels declined gradually after 40 days. No anaphylactic reactions, changes in core body temperature, or other adverse events were observed in the mice over the course of these injections of phage.

\section{Discussion}

The emergence of antibiotic-resistant bacteria, such as many IMPR-Pa and MDR-Pa that have spread over several continents, has highlighted the need to explore the potential therapeutic applications of alternative therapies, such as the utilization of bacteriophages. Although phages were discovered nearly 90 years ago, phage therapy as an antibacterial approach is still stagnant in the world. Experimental studies of phage therapy against MRSA and VRE bacteremia in mouse models were reported in recent years $(16,18)$, but little is known 
about phage therapy for $P$. aeruginosa, especially IMPR-Pa infections. Abul-Hassan and co-workers (13) used phages to control $P$. aeruginosa infection in 30 cases of resistant infections in patients with pseudomonas burn wound sepsis, only 18 cases of the 30 patients achieved satisfactory effect, and they concluded that it is not practical to use phage therapy on a wide scale and it should be restricted to cases with strains resistant to available antibiotics. A serial experiments conducted by Soothill $(12,14)$ in the 1990 s confirmed the validity of phage therapy in $P$. aeruginosa infection mouse models. However, basic features of the phages used for individual bacterial pathogens, including the pharmacokinetics involved, are still largely unclear. Moreover, early experiments were not well designed and the phage dosages used were not purified. For verification of the scientific as well as clinical validity of the therapy, well-characterized and purified phages would be desirable for therapeutic use.

In this study, using newly isolated lytic phages, we developed an animal model to determine the potential use of the phage to cure IMPR-Pa bacteremia. The results obtained in the present experiments are encouraging: after i.p. injection of MLD ( $\left.3 \times 10^{7} \mathrm{CFU}\right)$ dosage of IMPR-Pa bacterial strains from one side of the abdomen, i.p. injection of the phages at MOI $\geq 0.01$ from the other side rescued $100 \%$ of bacteremic mice. Even if phage treatment was delayed for up to $3 \mathrm{~h}$, approximately $40 \%$ of the animals were rescued and went on to recover completely. Heat-inactivated phages displayed no effect in the experiments, as compared to the therapeutic effect of active phages. The 'cocktail' of phage ØA392 and phage Ø1093 rescued bacteremic mice from IMPR-Pa 9747, 1613 and 9915, but it did not work for IMPR-Pa 9994 infection. Finally, phage particles were found in the circulation shortly after i.p. injection (within $2 \mathrm{~h}$ ) and they remained at a relatively high titers for $48 \mathrm{~h}$ or longer.

These experiments demonstrate a powerful curative effect of phages on IMPR-Pa bacteremia in our mouse model. The survival rate between phage-treated and the control groups is statistically significantly different. The survival rate of bacteremic mice by delayed treatment was reduced, suggesting that stressed animals are more sensitive to various factors, in this case either the phage itself or trace amounts of endotoxins and exotoxins present in the phage preparations. Healthy animals did not display apparent reactions to these factors, as evidenced by the lack of any adverse effects in the control groups inoculated with a high dose of the phage preparation.

Our studies uncovered that the efficacy of phage therapy in the rescue of bacteremic animals was due to a functional capability of the phage and not a non-specific immune effect, since rescue was not observed with heat-inactivated phage. In addition, by comparing the susceptibility of the bacterial strains to the phage mixture, we showed that the rescue of IMPR-Pa bacteremic mice was limited to 9747, 1613, and 9915 , indicating only those virulent phages that are capable of growing in and lysing the infecting bacterial strain in vitro are effective in the rescue of animals that are bacteremic with that bacterial strain. These data are consistent with the observations by Biswas et al (16). Furthermore, Matsuzaki and colleagues (18) confirmed that a 'mechanical' lysate of Staphylococcus aureus induced no antibacterial effect and phages had no therapeutic effect against infections with the phage-lysogenic host bacteria. On the other hand, bacterial antigens (such as endo- and exotoxins or lysins) should have been completely removed from the phage ØA392 preparations through repeated $\mathrm{CsCl}$-gradient centrifugation purification (15), and this may rule out the involvement of the body immune response in the rescue of the diseased animals. This conclusion was also supported by the fact that the levels of the antibody against the phage were not substantially elevated at the time when the bacteremic mice were protected by the phage.

Further studies also demonstrated that phage ØA392 rapidly entered into the circulation (within $2 \mathrm{~h}$ ) when administered intraperitoneally, indicating a rapid systemic distribution of the phage in vivo. Matsuzaki et al (18) and Merril et al (19) even found that phage particles were detected in different tissues and/or organs. But Merril et al (19) found a disadvantageous result. That is, circulating phages were captured by the splenic reticuloendothelial system and were rapidly eliminated from the blood. However, Merril et al (19) also reported that a small population of mutant $\lambda$ phages survived in the circulation, with a concomitant alteration to major head protein E. Therefore, the authors postulated that such 'serially passed' immune-escape mutants may facilitate improvements to the therapeutic efficacy of phages (19). In our study, infectious ØA392 existed at substantially high levels in the bloodstream. The titers of phage ØA392 in the circulation were $\sim 10^{4} \mathrm{PFU} / \mathrm{ml}$, and the phage was maintained at relatively higher titers until $48 \mathrm{~h}$ after injection. We found that i.p. injection of phages did not evoke intense immune reactions. Our observation and those of others (16,20-22) support the view that the immunologic elimination of phages in vivo may not be a serious issue for practical applications of phage therapy because before the occurrence of immunologic elimination in the body, the treating effect of phages has already been completed.

Altogether, our current study reinforces the view that phage therapy can be used to treat drug-resistant bacterial infections. To search for and characterize further phages with therapeutic potential may cast new light on treating diverse bacterial infectious diseases that are uncontrollable by currently available antibiotics.

\section{Acknowledgements}

This work was supported by Cheng-guang Projects for Young Scientists from the Department of Science and Technology of Wuhan, China (No. 20025001026 to J.H.), and by a grant from the National Institutes of Health, Bethesda, MD 20892, USA (No. P20RR16440-010003 to Q.Q.L).

\section{References}

1. Cohen ML: Epidemiology of drug resistance: implications for a post-antimicrobial era. Science 257: 1050-1055, 1992.

2. Cohen ML: Antimicrobial resistance: prognosis for public health. Trends Microbiol 2: 422-425, 1994.

3. Clark NM, Hershberger E, Zervosc MJ and Lynch JP: Antimicrobial resistance among gram-positive organisms in the intensive care unit. Infect Dis 9: 403-412, 2003.

4. Sulakvelidze A, Alavidze Z and Morris JG: Bacteriophage therapy. Antimicrob Agents Chemother 45: 649-659, 2001.

5. Kutter E: Phage Therapy: Bacteriophages as Antibiotics. Evergreen State College, Olympia, WA, 1997. 
6. Stone R: Stalin's forgotten cure, news focus. Science 298: 728-731, 2002.

7. Travis J: Viruses that slay bacteria draw new interest. Science News 157: 358-360, 2000 .

8. Smith HW and Huggins MB: Successful treatment of experimental Escherichia coli infections in mice using phage: its general superiority over antibiotics. J Gen Microbiol 128: 307-318, 1982.

9. Smith HW and Huggins MB: Effectiveness of phages in treating experimental Escherichia coli diarrhoea in calves, piglets and lambs. J Gen Microbiol 129: 2659-2675, 1983.

10. Smith HW, Huggins MB and Shaw KM: Factors influencing the survival and multiplication of bacteriophages in calves and in their environment. J Gen Microbiol 133: 1127-1135, 1987.

11. Smith HW, Huggins MB and Shaw KM: The control of experimental Escherichia coli diarrhoea in calves by means of bacteriophages. J Gen Microbiol 133: 1111-1126, 1987.

12. Soothill JS: Treatment of experimental infections of mice with bacteriophages. J Med Microbiol 37: 258-261, 1992.

13. Abul-Hassan HS, El-Tahan K, Massoud B and Gomaa R: Bacteriophage therapy of Pseudomonas burn wound sepsis. Ann MBC 3: 1-4, 1990.

14. Soothill JS: Bacteriophage prevents destruction of skin grafts by Pseudomonas aeruginosa. Burns 20: 209-211, 1994.

15. Sambrook J, Fritsch EF and Maniatis T: Molecular Cloning: A Laboratory Manual. Vol. 1. 2nd edition. Cold Spring Harbor Laboratory Press, Cold Spring Harbor, New York, pp66-79, 1989.
16. Biswas B, Adhya S, Washart P, Paul B, Trostel AN, Powell B, Carlton R and Merril1 CR: Bacteriophage therapy rescues mice bacteremic from a clinical isolate of Vancomycin-resistant Enterococcus faecium. Infect Immun 70: 204-210, 2002.

17. Adams KH: Bacteriophages. Wiley-Interscience, New York, 1959.

18. Matsuzaki S, Yasuda M, Nishikawa H, Kuroda M, Ujihara T, Shuin T, Shen Y, Jin Z, Fujimoto S, Nasimuzzaman MD, Wakiguchi H, Sugihara S, Sugiura T, Koda S and Muraoka A: Experimental protection of mice against lethal Staphylococcus aureus infection by novel bacteriophage ØMR11. J Infect Dis 187: 613-624, 2003

19. Merril CR, Biswas B, Carlton R, Jensen NC, Creed GJ, Zullo S and Adhya S: Long-circulating bacteriophage as antibacterial agents. Proc Natl Acad Sci USA 93: 3188-3192, 1996.

20. Dean JH, Silva JS and McCoy JL: In vitro human reactivity to staphylococcal phage lysate. J Immunol 115: 1060-1064, 1975.

21. Carlton RM: Phage therapy: past history and future prospects. Arch Immunol Ther Exp 47: 267-274, 1999.

22. Weber-Dabrowska B, Mulczyk M and Gorski A: Bacteriophages as an efficient therapy for antibiotic-resistant septicemia in man. Transplant Proc 35: 1385-1386, 2003. 